Meta

Journal des traducteurs

Translators' Journal

\title{
Recherches en traductologie en République fédérale d'Allemagne : tendances et perspectives
}

\section{Frank G. Königs}

Volume 31, numéro 2, juin 1986

URI : https://id.erudit.org/iderudit/003337ar

DOI : https://doi.org/10.7202/003337ar

Aller au sommaire du numéro

Éditeur(s)

Les Presses de l'Université de Montréal

ISSN

0026-0452 (imprimé)

1492-1421 (numérique)

Découvrir la revue

Citer cet article

Königs, F. G. (1986). Recherches en traductologie en République fédérale

d'Allemagne : tendances et perspectives. Meta, 31(2), 119-136.

https://doi.org/10.7202/003337ar d'utilisation que vous pouvez consulter en ligne. 


\title{
RECHERCHES EN TRADUCTOLOGIE EN RÉPUBLIQUE FÉDÉRALE D'ALLEMAGNE : TENDANCES ET PERSPECTIVES*
}

FRANK G. KÖNIGS

UNIVERSITE DE BOCHUM

\begin{abstract}
Und was soll ich viel und lange reden vom Dolmetschen? Sollt ich aller meiner Worte Ursachen und Gedanken anzeigen, ich müßte wohl ein Jahr dran zu schreiben haben. Was Dolmetschen für Kunst und Arbeit sei, das hab ich wohl erfahren; darum will ich keinen Papstesel noch Maulesel, die nichts versucht haben, hierin als Richter oder Tadler dulden. Wer mein Dolmetschen nicht will, der laß es anstehen. Der Teufel danke dem, der es nicht mag oder ohn meinen Willen und Wissen meistert. Soll's gemeistert werden, so will ich's selber tun. Wo ich's selber nicht tu, da lasse man mir mein Dolmetschen mit Frieden une mache ein jeglicher, was er will. für sich selbst und lebe wohl!

Martin Luther, "Sendbrief vom Dolmetschen (1530)", in : Störig, H.J. (ed.) (1963) : Das Problem des Übersetzens, Darmstadt : Wissenschaftliche Buchgesellschaft, pp. 14-32, ici p. 24.
\end{abstract}

\section{PRÉLIMINAIRES}

0.1 Il est bien osé et risqué, si ce n'est illusoire, que de vouloir, dans le cadre d'un article, se lancer à présenter ne serait-ce que de manière modestement adéquate les résultats des recherches ayant vu le jour, ou tout simplement encore en cours, dans un domaine scientifique représenté par l'ensemble des savants d'un pays. Automatiquement, on se voit contraint à instaurer des priorités ayant pour conséquence inévitable que l'un ou l'autre point de départ ou bien que l'un ou l'autre chercheur ne soit pas pris en considération de manière satisfaisante, voire même ne soit pas pris en considération. Ceci étant, dans les propos qui vont suivre je ne me suis pas assigné pour but de me livrer à une présentation vaste et complète de la situation en Allemagne. Bien au contraire, je vise une appréciation subjective de ces travaux de recherche dont je pense qu'ils ont exercé, qu'ils exercent encore, ou qu'ils sont susceptibles d'exercer une influence considérable dans le domaine de la traductologie. Bien entendu ce choix - le fruit d'une approche délibérément subjective - se doit d'être présenté en ses divers éléments de la manière la plus rigoureusement objective qui puisse être.

0.2 'Les sciences de la traduction' sont tout à fait dans la tradition allemande, tradition qui ne se retrouve pas seulement à l'actif de la recherche mais encore dans l'importance considérable accordée à la formation des traducteurs et interprètes. C'est ainsi que des programmes de formation pour traducteurs et interprètes ont vu le jour dans différents endroits avec des priorités en partie identiques, en parties différentes, mais tous des programmes de formation qui ont tous depuis longtemps dépassé la phase d'expérimentation et sont devenus partie intégrante de l'enseignement universitaire. Bien que la traductologie d'une part, et la formation des traducteurs et interprètes d'autre part, entretiennent d'étroits rapports entre elles, je me propose toutefois de mettre entre 
parenthèses les programmes de formation, ou tout au moins tout ce qui touche à l'adaptation relative aux curricula ainsi que les problèmes et expériences y ayant trait.

0.3 Lorsque dans le titre et dans les propos qui vont suivre il est question de l'Allemagne ou de la recherche en Allemagne, cela signifie que je me limite et me réfêre exclusivement aux travaux ayant vu le jour ou poursuivis actuellement en République fédérale d'Allemagne. Les travaux de recherche effectués ou actuellement en cours en République démocratique allemande ne seront pas retenus. Ceci n'a, bien entendu, rien à voir avec un éventuel manque d'intérêt de ma part, bien au contraire ! Toutefois la traductologie qui s'est développée en Allemagne de l'Est a à son actif des points de départ et des théories qui leur sont bien spécifiques. Autrement dit l'espace limité dont je dispose ici ne saurait me permettre de mentionner ces travaux, leurs mérites, leurs problèmes, ne serait-ce même qu'en passant.

C'est également pour des raisons d'ordre strictement matériel que je me vois contraint à renoncer dans les développements qui vont suivre tant à la présentation des problèmes posés par l'interprétariat qu'à une étude des problèmes relatifs à la traduction littéraire.

\section{LA TRADUCTOLOGIE EN TANT QUE DISCIPLINE SCIENTIFIQUE}

Contrairement aux usages langagiers propres à d'autres langues, il est presque devenu évident de parler en allemand d'une science de la traduction lorsqu'il s'agit de désigner un système de réflexions méthodiques relatives aux questions de la traduction comme processus d'une part, et comme produit fini d'autre part. Il est fort probable que ce phénomène provienne de ce besoin (typiquement germanique?) de se créer un ensemble ordonné, qu'il soit le fait de l'engagement et de l'intérêt de chercheurs isolés ou bien encore le fait d'une étroite mise en relation délibérément voulue entre l'enseignement universitaire et le domaine de recherche scientifique existant ou enfin qu'il soit le fait d'un intérêt porté à la traduction, intérêt dont la continuité se trouve fondée historiquement. Quoi qu'il en soit ce phénomène nous épargne ces discussions qui semblent nécessairement inévitables dans d'autres pays (cf. par exemple la présentation de Larose 1984). Que la traductologie constitue un domaine scientifique à part entière, voilà qui depuis bien longtemps déjà ne fait plus l'ombre d'un doute (cf. à titre d'exemple les titres de Bausch 1970 ; Wilss et Thome 1974a, 1974b ; Wilss 1977 ; Koller 1979 ; Stein 1980). Il est par contre un autre et brûlant sujet de discussion - les titres cités ci-dessus en témoignent - à savoir : quelle est l'orientation concrète que cette discicipline doit prendre, quels contenus et pour ceux-ci avec quelles priorités et dans quelle direction doitelle s'imposer. Sur un plan de pure organisation également, la traductologie passe pour science établie en raison notamment de l'existence d'une section portant le même nom dans le cadre de l'Association allemande de linguistique appliquée. magne?

Comment se présente donc, en sa qualité de discipline, la traductologie en Alle-

$\hat{A}$ en croire Koller $(1979: 10)$ elle traverserait une crise de légitimation et ceci, tant pour ce qui est des rapports qu'elle entretient avec la pratique même de la traduction qu'elle prétend refléter. Cette crise de légitimation se manifeste - toujours d'après Koller - dans la diversité des approches choisies pour résoudre certains problèmes et dans les priorités adoptées respectivement selon l'intérêt en jeu, priorités déterminant chaque fois l'étude de questions d'ordre scientifique et relatives à la traduction. On peut avoir des avis divergents quant à la question de savoir s'il est vraiment souhaitable de viser, à l'intérieur d'une discipline scientifique, à une autorestriction instaurant une seule et unique méthode de recherche et un seul et unique catalogue de questions classées se- 
lon une certaine hiérarchie. Il me semble que c'est précisément un avantage des disciplines lorsqu'elles comportent une littérature thématique très riche, car cela prouve que de nombreux chercheurs se penchent sur des problèmes analogues, mais en adoptant des perspectives en partie différentes et, à mon avis, c'est précisément ce fait qui permet de promouvoir une discipline au rang de domaine de recherche établi. C'est la discussion qui anime l'affaire, et la traductologie ne saurait en aucun cas se plaindre d'une pénurie de discussion, surtout pas en Allemagne. Par contre, l'autre phénomène mentionné par Koller à titre d'indice révélateur d'une crise de légitimation doit être déjà plus pris au sérieux : la traductologie - de quelque orientation qu'elle soit - se doit de prétendre être directement en prise sur la pratique. Ceci est une condition sine qua non reposant sur l'intérêt scientifique même de la traductologie, à savoir être en mesure de livrer des résultats et des acquis sur l'activité pratique de la traduction. Il me semble tout à fait symptomatique pour la traductologie en Allemagne (mais pas seulement en Allemagne) que ses approches théoriques n'aient pas toujours pour but l'exercice de la pratique et qu'elles soient, de temps à autre, pour le praticien ni intéressantes ni compréhensibles ${ }^{1}$. On peut, à mon sens, compenser cette défectuosité bien moins par un monisme et une restriction des perspectives que par un resserrement des liens entre les réflexions d'ordre théorique et les phénomènes et les conditions propres à la pratique (cf. Hönig et Kussmaul 1982; Königs 1985a).

Contrairement à Koller, Wilss $(1977 ; 1980$ : 9sq.) souligne - et de façon positive - le fait que la traductologie trouve l'objet de son intérêt scientifique dans des processus susceptibles de s'effectuer aussi bien dans le cadre d'une étroite comparaison entre deux langues que dans celui d'une comparaison plus générale, c'est-à-dire ne s'appuyant pas sur la prise en considération de langues. Pour cette raisōn, la traductologie reconnaît comme impératif le recours inéluctable tant à la linguistique qu'à la psycholinguistique et à la sociolinguistique. Reste à savoir - et ce précisément dans le cas de l'Allemagne - si elle l'a toujours fait. La plupart du temps - et non sans quelque succès assurément - elle s'est 'appuyée' sur la linguistique et lui a emprunté également de temps en temps, outre des méthodes descriptives, sa façon de poser des hypothèses heuristiques propres à engendrer l'investigation scientifique (cf. Reiss 1971, 1976; Reiss et Vermeer 1984 ; Diller et Kornelius 1978 ; aussi Albrecht 1973). Que cela ne soit toutefois pas suffisant compte tenu de l'intérêt scientifique propre à la traductologie, que cela puisse même entraîner une réduction tout à fait récusable de la perspective adoptée, c'est ce que Stein (1980) et Königs (1979 ; 1985a ; 1985b ; 1986) ont essayé, avec d'autres, de démontrer.

Un trait caractéristique de la traductologie en Allemagne se révèle être à mon avis le fait que l'on puisse la répartir selon des angles d'investigation différents, déterminés chacun en fonction des priorités que les chercheurs concernés ont adoptées pour leur recherche ; ceci, bien entendu, n'exclut pas que quelques 'chefs de file' travaillent soit simultanément, soit successivement dans plusieurs perspectives. De temps à autre ces perspectives se recoupent, mais s'excluent réciproquement selon les cas, si bien que l'on devrait à coup sûr éviter de parler de classification.

\section{LA PERSPECTIVE PROPRE À L'ANALYSE DE TEXTE}

Il semble évident, et cela permet de se dispenser de toute autre explication, que le texte (de départ) est et doit être de première importance dans toutes les perspectives de la recherche en traductologie. Toutefois des différences peuvent se constater quant à la question de la primauté de l'importance accordée au texte (de départ) dans l'optique du processus de traduction. Ceci dit, la marque typique de la perspective propre à l'analyse de texte est que l'essentiel des réflexions tourne autour de la question suivante : Comment le texte de départ peut-il se laisser décrire, linguistiquement parlant, afin de facili- 
ter ainsi la traduction comme activité, de rendre la traduction comme résultat de cette activité plus transparente ? D'importants représentants de cette perspective qui puisent leurs 'impulsions' dans la 'linguistique de texte' visent, dans leurs travaux, à une systématisation des opérations linguistiques à la surface des textes de départ, systématisation à partir de laquellle ils recherchent une transposition dans la langue cible (cf. Dressler 1972a ; Dressler 1972b ; Thiel 1974 ; Thiel 1981; cf. aussi Albrecht 1973 et Diller et Kornelius 1978). Cette façon de procéder signifie d'une certaine manière un développement plus avancé de la stylistique comparée telle qu'elle a été fondée et pratiquée par Vinay et Darbelnet (1958) et Malblanc (1962). La poursuite de cette évolution consiste d'une part dans la prise en considération d'une spécificité relative au type de texte (cf. Thiel 1981) ainsi que dans la comparaison des types de texte (cf. Reiss 1977; Spillner 1981) du point de vue de la traduction, d'autre part dans l'adaptation aux courants de linguistique textuelle chaque fois actuels (par exemple Beaugrande et Dressler 1981). La critique majeure que l'on puisse formuler à l'égard de cette approche propre à l'analyse de texte est celle d'un manque de concomitance avec la réalisation même du processus de traduction. En fait, les indices sont quasi inexistants pour ce qui est de prouver que le traducteur (qu'il s'agisse de l'étudiant ou du traducteur chevronné) procède effectivement d'une telle manière analytique et ce, comme les représentants de cette perspective le supposent. Il se peut, bien sûr, que l'analyse du traducteur dans des cas isolés se déroule de façon analogue à ces idées relatives à un modèle d'analyse, mais il se peut fort bien également qu'elle suive, cette analyse, d'autres critères scientifiques de classification (tels par exemple que le facteur temps, le commettant, la 'qualité', le destinataire, l'expérience personnelle). D'un point de vue général, la perspective propre à l'analyse de texte est, assurément, celle qui doit le plus à la linguistique. Ceci dit, il ne faudrait pas omettre de noter qu'elle a, pour une multitude de questions relatives à telle ou telle langue, suscité une prise de conscience générale des problèmes et qu'elle a ainsi souligné la nécessité de mettre à jour des manières générales de procéder lors de la traduction.

\section{LA PERSPECTIVE HERMÉNEUTIQUE}

Tandis que la perspective propre à l'analyse de texte envisage essentiellement le texte sur l'arrière-plan de sa rédaction structurelle et syntactique, ce dernier va, dans le cadre de la perspective herméneutique, se trouver bien plus étroitement assujetti au 'macro-contexte'. Dans ce cas, des traits caractéristiques d'ordre structurel se trouvent, quant à leur importance, relégués au second plan par rapport à la compréhension globale. L'essentiel pour les représentants de cette perspective herméneutique (cf. Paepcke et Forget 1981 ; Stolze 1982) est l'intention qui a présidé à la rédaction du texte, c'est-àdire ce vers quoi tend le texte en tant que message. Dans ces conditions les composantes formelles et structurelles du texte se trouvent tout simplement reléguées au rang d'éléments constitutifs de l'idée à exprimer et non plus comme des éléments constitutifs du texte jouissant jusqu'à un certain point d'une certaine autonomie. L'interaction du contenu et de la forme, objet par excellence de la pragmatique linguistique, est cependant laissée pour compte en raison de l'ambition 'macro-contextuelle' de la perspective herméneutique. Qu'il me soit permis de citer ici les propos suivants à titre d'exemple typique pour la perspective herméneutique :

En résumé, la conscience herméneutique accepte de se placer dans la réflexion elle-même. Son effort ne consiste pas à objectiver la compréhension, mais à la désubjectiviser au maximum tout en acceptant l'aporie, à la manière du récitant faisant la lecture d'un poème et cherchant à évacuer le plus possible ce qui n'est pas dans le texte qu'il lit, à savoir la contingence que représentent sa voix et sa diction, pour approcher l'idéalité du texte. Mais si cette irréductible contingence heurte l'idéalité du texte, elle est aussi constitutive de l'existence que le récitant donne au poème par sa voix, de son interprétation et donc de sa compréhen- 
sion du texte, et l'aporie initiale se retourne pour devenir positive car créatrice. De la même manière, on voit mieux maintenant en quoi la traduction est un modèle privilégié de la conscience herméneutique en ce qu'elle s'approprie un autre tout en reconnaissant l'altérité. La traduction ne se réduit pas à l'obsession identificatrice que dévoile l'idéal naïf du mot à mot comme son opposé, l'idéal annexionniste. Le traducteur fait se rejoindre l'autre avec lui-même (Horizontverschmelzung) tout en reconnaissant la présence de l'autre en luimême : sa conscience est toujours en même temps solidaire et solitaire. (Paepcke et Forget 1981 : 24f.)

An die Stelle der den Naturwissenschaften eigenen Objektivität tritt die vorgängige Beziehung zum Gegenstand des Textes, und an die Stelle der objektiven Erkenntnis tritt das Ideal der Teilhabe. In der Erfahrung dieser Grösse, die ïbersubjektiv ist, liegt das Objektive der Hermeneutik. Zwar kommt der Text nur durch Interpretation zum Sprechen; aber wie bei einem sachlichen Gespräch, das ja, wenn es zur Verständigung in der Sache führt, also dem Gegenstand unter einer bestimmten Fragestellung angemessen ist, gerade nicht subjektiv, sondern objektiv genannt wird, gehen die eigenen Vormeinungen des Interpreten nicht in die Auslegung selbst ein, sondern wirken mehr wie eine Meinung, die man in der Diskussion aufs Spiel setzt, um den Gegenstand herauszuarbeiten. Diese eigenen Vormeinungen sollen dabei nicht abgebaut oder ausgeblendet, sondern reflektiert werden (...). Die Schwierigkeit liegt dabei in der Fremdheit des Textes. Es gilt, zunächst die Überlegenheit des Textes im Mitteilungsgeschehen (...) anzuerkennen und auszuhalter. (Stolze 1982 : 195.)

Le reproche majeur que l'on formule et que l'on se doit de formuler - et ceci pas tout à fait à tort - à l'égard de la perspective herméneutique à côté d'autres reproches ${ }^{2}$ consiste à dire qu'il justifie son existence de par sa prétention à offrir des critères d'appréciation 'objectifs' pour le texte et la traduction alors qu'il rend plutôt confus ces critères d'appréciation et qu'il s'éloigne tellement des conditions pratiques de la traduction comme activité que sa valeur d'application s'en trouve extrêmement réduite. C'est pourquoi il n'y a rien de surprenant à ce que de nombreux résultats de traduction apparaissent comme étant 'reconstructibles', ce qui toutefois n'est pas le cas pour les justifications parfois longues et (pseudo-)philosophiques (cf. Paepcke et Forget 1981) invoquées à cet effet. Ces dernières mettent par ailleurs en évidence que les notions structuralistes ainsi que les éléments théoriques qui s'y attachent ne sont pas aussi inadéquats que les représentants de la perspective herméneutique veulent bien le croire. De ceci, il ressort clairement qu'une position extrême, celle notamment de la théorie de la traduction comme théorie linguistique, est tout aussi insoutenable que l'autre. Autrement dit : sans linguistique aucune, cela ne va pas non plus.

\section{LA PERSPECTIVE DE LA TRADUCTION CRITIQUE}

À l'opposé de la perspective herméneutique, dont l'ambition a tout aussi bien pour objet le processus de la traduction que le texte traduit (c'est-à-dire le résultat de la traduction) et qui revendique ainsi la même valeur aux yeux du traducteur qu'à ceux de celui qui doit juger d'un texte traduit, la perspective de la traduction critique, quant à elle, s'intéresse en premier lieu uniquement au résultat obtenu par la traduction. Elle veut livrer des critères qui rendent possible une évaluation des textes traduits et plus précisément une évaluation libre de tous hasards, style feuilleton et qui se laissent vérifier de manière générale. Tandis que l'approche herméneutique est orientée vers la prospective dans la mesure où son point de départ est le texte de départ lui-même qui, seul, lui permet de viser le texte cible, l'approche de la traduction critique, elle, procède - et ce traditionnellement - de manière rétrospective : son point de départ étant le résultat, le texte traduit, et sa visée étant alors de permettre à celui qui est amené à formuler des critiques, de tirer des conclusions quant à la conception que le traducteur s'est faite du texte de départ. Par conséquent, la traduction critique s'est tout d'abord préoccupée de 
questions relatives à une typologie de texte pertinente pour la traduction. Ceci se trouve tout particulièrement réalisé dans les travaux de Katharina Reiss : Pour elle, une " typologie de texte respectant les impératifs du processus de la traduction et susceptible de s'étendre à toutes les sortes de textes présents dans la pratique de la traduction est la condition préalable indispensable permettant la pratique d'une critique objective de la traduction " (Reiss 1971 : 24). En s'appuyant sur le Modèle Organon de Bühler, elle établit tout d'abord la distinction entre le texte fidèle au contenu, le texte fidèle à la forme, le texte fidèle quant aux effets recherchés et le texte obéissant aux règles du genre (Reiss 1971). Elle décrit ces différents types de texte sur l'arrière-plan d'instructions et de régularités propres à une langue tout en tenant compte des facteurs paralinguistiques (rapport concret, rapport temporel, rapport local, rapport quant au destinataire, dépendance liée au locuteur, implications affectives). Ainsi se trouve impliqué, ne serait-ce que sous la forme d'allusion, la dimension pragmatique de la langue en général et par conséquent du phénomène de traduction en particulier. En même temps, il apparaît clairement que la traduction comme phénomène et la critique de la traduction ne sauront jamais être objectives au sens absolu du terme. Et c'est également sous cet angle que l'approche de la traduction critique de Reiss se trouve en opposition avec la prise de position herméneutique. Dans un travail ultérieur Reiss (1976) parfait son approche et, ce faisant, esquisse le schéma suivant fort bien choisi pour représenter son modèle :

\begin{tabular}{|c|c|c|c|c|}
\hline $\begin{array}{l}\text { Type } \\
\text { de texte }\end{array}$ & $\begin{array}{l}\text { Fonction } \\
\text { du texte }\end{array}$ & Particularité & $\begin{array}{l}\text { Principe } \\
\text { d'équivalence }\end{array}$ & $\begin{array}{l}\text { Méthode de } \\
\text { traduction } \\
\text { fonction } \\
\text { primaire } \\
\end{array}$ \\
\hline 1. informatif & $\begin{array}{l}\text { transmission } \\
\text { d'informations }\end{array}$ & $\begin{array}{l}\text { adapté au } \\
\text { contenu }\end{array}$ & $\begin{array}{l}\text { invariabilité } \\
\text { quant au } \\
\text { niveau du } \\
\text { contenu }\end{array}$ & $\begin{array}{l}\text { conforme au } \\
\text { contenu } \\
(=\text { tout } \\
\text { simplement } \\
\text { prosaïque) } \\
\end{array}$ \\
\hline 2. expressif & $\begin{array}{l}\text { message } \\
\text { artistique }\end{array}$ & $\begin{array}{l}\text { adapté à } \\
\text { l'émetteur du } \\
\text { message }\end{array}$ & $\begin{array}{l}\text { analogie dans } \\
\text { la mise } \\
\text { en forme } \\
\text { artistique }\end{array}$ & $\begin{array}{l}\text { conforme à } \\
\text { l'originalité } \\
\text { de l'auteur } \\
\text { (= recherche } \\
\text { d'identification) }\end{array}$ \\
\hline 3. opérationnel & $\begin{array}{l}\text { déclenchement } \\
\text { de } \\
\text { comportements }\end{array}$ & $\begin{array}{l}\text { adapté en } \\
\text { fonction du } \\
\text { comportement } \\
\text { visé }\end{array}$ & $\begin{array}{l}\text { identité de } \\
\text { l'appel } \\
\text { immanent } \\
\text { au texte }\end{array}$ & $\begin{array}{l}\text { conforme à } \\
\text { l'appel contenu } \\
\text { dans le texte } \\
\text { (parodie puis } \\
\text { adaptation) }\end{array}$ \\
\hline $\begin{array}{l}\text { 4. audio- } \\
\text { diffusé et } \\
\text { audio-visuel }\end{array}$ & $1-3$ & $1-3$ & $1-3$ & $\begin{array}{l}\text { conforme au(x) } \\
\text { médium (mé- } \\
\text { dia) utilisé(s) } \\
\text { (= supplétif) }\end{array}$ \\
\hline
\end{tabular}

Reiss $(1976: 20)$

Ceci dit, l'approche de Reiss a provoqué diverses critiques, et ce pour des raisons bien différentes. Tandis que Königs (1979 : 34 sq.) se préoccupe essentiellement de la question relative à la compétence effectivement susceptible d'être présupposée chez le 
traducteur et du problème de l'application pratique du modèle dans l'expérience quotidienne (Königs 1985a), Koller (1978; 1979 : 196 sqq.) critique tout particulièrement la présupposition implicite d'une norme assez rigide sans laquelle le modèle n'est pas fonctionnel, le manque de clarté concernant la définition de la notion de 'type de texte' ainsi que celle de 'méthode de traduction', la difficulté relative à une classification précise des textes selon cette typologie et surtout la non prise en considération du fait que les textes se situent toujours dans des rapports de communication bien précis. Dans toute une série d'exemples concrets, Katharina Reiss a livré la démonstration de la fonctionnalité (tout au moins partielle) de son approche $(1977 ; 1978 \mathrm{a} ; 1978 \mathrm{~b} ; 1979 ; 1980 \mathrm{~b})$. Même si encore ici et là apparaissent aux yeux du spécialiste critique des défauts et des lacunes dans cette approche, il n'en reste pas moins la constatation que Katharina Reiss a livré des idées et des propositions qui ont fait progresser considérablement la discussion relative aux questions posées par l'approche critique de la traduction, et qu'elle a ainsi posé les jalons pour de nombreuses impulsions nouvelles profitables à la traductologie. Ceci étant, on peut également retenir que son approche mène bien plus loin que la proposition de Koller (1979 : 210ff.) (tant pour ce qui est de la formulation que pour ce qui est de l'application pratique). En effet, Koller, quant à lui, répartit la critique scientifique de la traduction en analyse de texte pertinente pour la traduction, en étude comparative de la traduction et en évaluation de la traduction. Ce faisant, il commet dans la première partie la même 'faute' que celle qu'il reproche à Katharina Reiss, à savoir le maintien d'une difficulté partielle quant à une classification effective correspondant au mode de distinction de textes qu'il a délibérément choisi d'une part et l'adoption d'un niveau assez élevé comme critère censé correspondre à l'usage d'autre part. Par contre, les propos qu'il tient en matière d'étude comparative de la traduction ainsi qu'en matière d'évaluation de la traduction sont beaucoup trop généreux pour pouvoir contenir des suggestions concrètes permettant le passage à la pratique. À ce point de vue, le modèle de Reiss peut se réjouir d'une plus grande portée.

Outre les travaux de Reiss, le modèle de Juliane House (1977) représente une contribution non négligeable ayant dans une ample mesure influencé la discussion dans le cadre de la perspective critique de la traduction. Alors que Reiss prend comme point de départ essentiellement le texte, donc la langue, l'originalité de House consiste à intégrer simultanément l'utilisateur de la langue ainsi que des caractéristiques langagières et l'emploi de la langue tel qu'il a lieu et tel qu'il est vérifiable d'une part et à faire de l'ensemble le point de départ de ses réflexions d'autre part, élaborant ainsi le schéma d'analyse suivant :

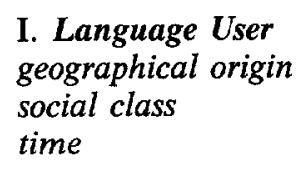

I. Language User geographical origin social class time

\author{
II. Language Use \\ medium \\ participation \\ social role relationship \\ social attitude \\ province
}

Contrairement au procédé rétrospectif traditionnel que l'on rencontre dans l'approche critique de la traduction, House opère de manière prospective dans la mesure où elle applique tout d'abord ses critères au texte de départ et aboutit ainsi à une analyse descriptive de ce texte de départ. À la suite de quoi elle passe alors, en se servant du même matériel instrumental, à la description du texte d'arrivée. Les écarts ainsi constatés constituent d'une part la base sur laquelle peut travailler la perspective de la traduction critique, d'autre part ils peuvent également fort bien être évalués de façon positive à travers la définition à chaque fois orientée de manière fonctionnelle des différents critè- 
res mis en œuvre. Autrement dit, House ne propose pas un schéma contraignant mais un modèle rendant compte des configurations pragmatiques et situationnelles de la langue, modèle qu'elle met elle-même à l'épreuve en lui soumettant elle-même pour servir d'exemple huit couples de textes choisis parmi les divers types de texte. On peut se permettre deux remarques critiques à l'égard de son modèle : d'une part certains critères utilisés ne se laissent pas différencier de manière satisfaisante les uns par rapport aux autres (ainsi par exemple : social class vs. social role relationship), d'autre part il n'est pas toujours possible de procéder à une classification objective irréprochable des éléments langagiers en diverses sous-classifications. Ceci dit, il est indubitable que le modèle de House représente, lui aussi, une contribution fructueuse et prometteuse à la discussion menée dans le cadre de la traductologie.

\section{LA PERSPECTIVE FONCTIONNELle}

La perspective fonctionnelle se trouve étroitement liée avec les travaux de la perspective critique de la traduction de Reiss et surtout de House. Le trait caractéristique de cette perspective fonctionnelle est que des équivalences statiques et par conséquent normatives donc restrictives, équivalences bien entendu entre langue de départ et langue d'arrivée, sont abandonnées ou évitées. Ainsi, le texte d'arrivée se trouve bien plus envisagé du point de vue de sa fonction communicative que du point de vue de son rapport avec des équivalences linguistiques formelles. L'oriéntation vers la fonction communicative ne prend pas simplement en considération le caractère conventionnel et déterminé socio-culturellement des textes (un texte allemand en langue de spécialité est construit différemment que son homologue anglais), mais aussi l'orientation adoptée par un texte traduit et ce en fonction de la commande faite. Aux côtés de House (1977) et de Reiss et Vermeer (1984), ce sont Hönig et Kussmaul (1982) qui ont représenté cette approche de la manière la plus marquante :

Je nach Auftrag erhalten wir so zwei ganz verschiedene Arten von Übersetzung - ohne Auftrag erhalten wir überhaupt keine Übersetzung. Wir sind der Meinung, dass es sich hier um zwei gleichberechtigte Grundtypen der Übersetzung handelt, die zwar hier auf Grund des AS-Textes [Ausgangssprachlicher Text, F.G.K.] in besonders pointierter Form vorgestellt werden, die aber trotzdem die erste und hierarchisch wichtigste Alternative darstellen, die mitiels einer Übersetzungsstrategie zu bewältigen ist.

Diese beiden Grundtypen lassen sich als Funktionskonstanz und als Funktionsveränderung bezeichnen. Es sind völlig gleichberechtigte und gleichermassen legitime Übersetzungsstrategien, zwischen denen der Übersetzer bei jedem Text zu wählen hat. Es ist also keineswegs so, dass Funktionskonstanz als Normalfall des Übersetzens gelten kann, während die Funktionsveränderung eine exotische Ausnahme darstellt.

(Hönig et Kussmaul $1982: 40$ )

Dans le cadre de cette perspective fonctionnelle on attache ainsi, en général, plus de valeur à l'intégration de la traduction dans l'ensemble communicatif de la langue d'arrivée qu'à une mise en équivalence $a$ priori, c'est-à-dire ignorant la situation, des éléments de la langue de départ et de la langue d'arrivée. Ceci signifie qu'une traduction peut/doit aboutir à un texte très différent, et ce, en fonction de la commande passée pour la traduction ; une appréciation absolue comme étant en tout cas fausse, en tout cas juste est par conséquent impossible - abstraction faite, bien entendu, des erreurs de grammaire commises dans la langue d'arrivée. C'est au traducteur qu'il revient d'orienter le procédé de traduction ${ }^{3}$ pour lequel il se décide finalement, s'attachant à la fonction visée et non pas, comme on le suppose souvent au texte lui-même et à son appréciation linguistique. 


\section{LA PERSPECTIVE RELATIVE DU PROCESSUS MÊME DE TRADUCTION}

Depuis très longtemps déjà, il est question, à l'intérieur de la traductologie, du processus de traduction lui-même. Cependant, la plupart du temps on utilise la notion de 'processus' tout simplement pour désigner ce qui se passe entre le texte de départ et la traduction du texte d'arrivée (cf. principalement les descriptions de Wilss 1977 et Koller 1979). Il faut reconnaître toutefois que Koller attire explicitement l'attention sur le fait que le processus de traduction ne saurait en aucun cas être ramené au processus mental. Ce processus quant à lui se pose comme objet de recherche pour la psycholinguistique d'une part et d'autre part se pose comme étant également extrêmement difficile pour ne pas dire impossible. Même si l'on est tenté d'approuver de prime abord cette argumentation traditionnelle dans la traductologie, une tout autre image se précise toutefois à un examen plus attentif. Il est absolument impossible de se passer de la psycholinguistique. Ce constat d'importance pour la traductologie future se déduit de deux observations fondamentales. L'une concerne les notions utilisées habituellement dans la traductologie. À côté de la notion de 'processus' apparaissent également continuellement les notions de 'stratégie' ou de 'difficulté' comme notions clés des réflexions théoriques. Ces notions jouent, entre autres, également un rôle important dans les théories psycholinguistiques, et c'est pourquoi il ne peut être que judicieux de ne plus ignorer plus longtemps la psycholinguistique avec ses procédés et ses résultats. Par ailleurs, il est à noter que des notions telles que celles de 'méthode', de 'procédé', de 'transfert' et d'unité' apparaissent également dans d'autres contextes relatifs aux sciences sociales. Il se peut que ce soit cette accumulation de notions étrangères à la discipline scientifique et de celles pourvues d'un sens plus général qui ait incité Wilss (1983) à essayer de délimiter conceptuellement l'une par rapport à l'autre la stratégie de la traduction, la méthodologie de la traduction et enfin la technique de la traduction.

La deuxième observation fondamentale intervenant en faveur de l'intégration de la psycholinguistique se situe au cœur même de l'activité de traduire elle-même. La validité du consensus suivant n'est plus à prouver : il n'existe pas et il ne saurait exister une seule et unique possibilité de traduction et divers traducteurs disposant de la même compétence de traduction n'aboutissent en général pas à un résultat qui serait strictement le même (cf. Reiss 1976; Hönig et Kussmaul 1982 ; Wilss 1983 ; Reiss et Vermeer 1984). En conséquence de quoi il est absolument indispensable tant pour la théorie scientifique de la traduction que pour la didactique de la traduction que l'on dispose d'informations confirmées relatives au 'quoi' et au 'comment', relatives à ce qui se produit entre le texte de départ et le texte d'arrivée.

Un premier abord possible de la perspective relative au processus même de la traduction se présente dans la preuve qui a été faite qu'une unité de traduction n'est pas définissable si on dépasse le niveau individuel (Königs 1981b). Bien entendu, elle se laisse décrire du point de vue linguistique, mais non pas à titre de procédé du traducteur, procédé obéissant à des descriptions linguistiques. Ce qui caractérise bien plus ce procédé ce sont, par contre, des éléments qui se trouvent transposés de manière ad hoc dans la langue d'arrivée (on parle de 'bloc ad hoc' et d'autres éléments qui ne sont transposés que peu à peu et de manière cognitive dans la langue d'arrivée (le 'bloc subsistant') (Königs $1981 \mathrm{~b} ; 1985 \mathrm{~b})$. En s'appuyant sur ce résultat que l'on a pu obtenir grâce à une analyse scientifique de la traduction, analyse largement traditionnelle, on pourrait entamer des réflexions quant à la question de savoir comment aborder les processus mentaux. La psychologie et la psycholinguistique offrent pour ce faire tout une série de procédés introspectifs à l'aide desquels il est possible d'obtenir quelque compréhension des processus mentaux (cf. par exemple Ericsson et Simon 1984 ; Huber et Mandl 1982 ; Wagner 
et al. 1981). Entre-temps, de tels procédés ont également été appliqués à l'activité de traduction. Ce faisant,

- l'hypothèse relative à l'existence de 'blocs $a d$ hoc' et de 'blocs subsistants' a été empiriquement confirmée,

- une assez grande résistance des éléments du 'bloc ad hoc' vis-à-vis de transformations possibles a été démontrée,

- l'importance toute particulière de mots essentiels pour la mise sur pied de la compréhension du texte imposée par la traduction a été mise en évidence (cf. Königs 1985a ; 1986).

Ceci dit, l'étude de la perspective relative au processus même de traduction mène inéluctablement à une discussion à propos de notions et de concepts tels que ceux de 'stratégie' ou bien encore de 'difficulté de la traduction'. À ce sujet c'est surtout Krings (1985 ; en préparation) qui argumente sur la base d'une étude empirique en faveur d'une notion 'mentale' de stratégie, notion qui se distingue fondamentalement de cette acception de notion de stratégie impliquant la réalisation progressive de phases de travail préalablement définies, acception que l'on trouve par exemple chez Hönig et Kussmaul (1982). En utilisant des procédés introspectifs, Krings esquisse une théorie systématique du processus de traduction, théorie dont l'association de valeurs réelles et de valeurs virtuelles est, en fin de compte, également d'une importance fondamentale pour la didactique de la traduction. Ce n'est que sur cet arrière-plan de l'intégration théorique (cf. Königs $1979: 9$ ) et surtout pratique (cf. Krings, en préparation) de cet agencement que constitue la compétence de traduction, de cette intégration donc dans le processus de traduction, que de nouveaux pas peuvent être franchis pour ce qui est d'une analyse de la traduction, analyse se rapportant aussi bien au texte qu'aux personnes concernées.

Même si parmi les recherches relatives à la traduction, celles qui sont orientées vers la psycholinguistique n'en sont qu'à leurs débuts, il apparaît déjà cependant que les modèles reposant sur des phases et à caractère statique tels qu'ils ont été présentés et discutés par Wilss $(1977 ; 1983)$ sont tout au plus d'une importance relative pour la formation d'une théorie ou bien alors qu'ils doivent être compris comme des modèles abstraits dont l'importance pour une traductologie appliquée, représentée tout particulièrement par Wilss (1981 : 19sq.), serait par contre à rediscuter. Ceci est surtout valable pour le cas où cette science appliquée de la traduction doit se trouver en rapport direct avec une traductologie se situant à un niveau général, c'est-à-dire indépendant de toute prise en considération de langues particulières.

\section{LA PERSPECTIVE DIDACTIQUE DE LA TRADUCTION}

Que doit viser une traductologie appliquée ? Outre la pratique professionnelle du traducteur, elle visera - surtout si elle s'appuie sur la perspective du processus même de traduction - l'enseignement et l'apprentissage de la traduction. Elle essaiera ainsi d'élaborer à partir de l'éventail extrêmement riche des approches possibles de la traduction (par exemple les différentes perspectives présentées ici), des recommandations relatives à l'acquisition et à la transmission de la compétence de traduction. Il en résulte pour la didactique de la traduction un champ complexe de tâches à accomplir. Il s'agira alors de faire la distinction entre 'la traduction comme compétence de traduire' et " la traduction comme type d'exercice ", et ceci - bien entendu - en raison du caractère spécifique propre à chacune de ces deux activités (Bausch 1977b). Par ailleurs, la didactique de la traduction est compétente pour ces deux types d'activité dans lesquels peut s'exercer la traduction, car la traduction dans le cadre de l'enseignement des langues étrangères et la traduction dans le cadre de la formation des traducteurs ont des points communs (que la psycholinguistique doit mettre à jour) et sont, en outre, à certains stades soumis à des objectifs d'apprentissage comparables (voir à ce sujet les contributions 
essentiellement centrées sur l'enseignement des langues étrangères dans les recueils de Bausch et Weller 1981 et actuellement de Titford et Hieke 1985 ainsi que certains articles visant essentiellement la formation des traducteurs dans Wilss et Thome 1984).

Tandis que cette branche de la didactique de la traduction se rapportant à l'enseignement des langues étrangères repose sur une tradition relativement longue ${ }^{4}$ se reflé- $^{-}$ tant dans la vive discussion débattant du pour et du contre de la traduction dans l'enseignement des langues étrangères (voir la bibliographie de Weller 1977), des réflexions concernant la didactique de la traduction dans le cadre de la formation des traducteurs n'est apparue que dans les dix dernières années (cf. par exemple Wilss 1975, 1978 ; Reiss 1976, 1980a ; Bausch 1977a, 1977b ; Königs 1979 ; Raabe 1979, ainsi que le recueil de Wilss et Thome 1984). Dans certains de ces articles la didactique de la traduction est envisagée comme une 'application' découlant linéairement de la théorie (linguistique) de la traduction. Le procédé que l'on a rencontré pendant des années dans le cadre de travaux relatifs à la didactique des langues étrangères a été - et ce pour des raisons d'ordre méthodologique - l'objet d'un rejet catégorique (voir à titre d'exemple Bausch 1979) et ceci pour une bonne part au nom de l'autonomie des lois régissant la réalité concrète de toute situation d'enseignement. De manière analogue, une mise à contribution de tous les facteurs impliqués dans la réalité propre à toute situation d'enseignement doit être exigée et reconnue légitime pour l'enseignement de la traduction dans le cadre de la formation des traducteurs, ainsi que pour les branches alors compétentes de la didactique de la traduction (cf. Königs 1985b ; 1986).

Comme tâches à accomplir pour une organisation conceptuelle de la didactique de la traduction on citera :

- la prise en considération systématique de résultats et de méthodes de recherche émanant de la psychologie, de la psycholinguistique, de la pédagogie, de la neurolinguistique et de l'observation de situations d'enseignement ;

- la tentative d'élaboration d'une esquisse théorique autonome ayant pour but d'intégrer à une théorie spécifiquement orientée vers l'enseignement de la traduction dans le cadre de la formation des traducteurs, d'intégrer donc à une telle théorie des éléments provenant de disciplines diverses mais intéressant directement l'objet de la recherche, et non point de se contenter de les juxtaposer les uns aux autres de manière éclectique;

- la prise en considération de la traduction dans le cadre de l'enseignement des langues étrangères aussi bien comme forme autonome de réalisation de la traduction qu'en comparaison avec la traduction telle qu'elle est réalisée dans l'enseignement destiné à la formation des traducteurs, et ainsi comme partie intégrante d'une théorie de la didactique de la traduction. duction :

Se présentent alors comme tâches concrètes à réaliser pour la didactique de la tra-

a) le recueil de constats réalisés dans la pratique de l'enseignement de la traduction (au niveau scolaire cf. Grotjahn et Klevinghaus 1975, au niveau secondaire cf. Königs 1979);

b) la classification systématique de types de tâches et ce, en fonction du contenu, du rôle et des exigences langagières (cf. la tentative de Weller 1981 relative à l'enseignement des langues étrangères);

c) la mise à l'essai de différentes manières (méthodologiques) de procéder (traduction au niveau de la phrase, au niveau du paragraphe, contre-épreuve, comparaison de traductions, diverses méthodes de travail sur le vocabulaire (cf. les aspects didactiques de la comparaison de la traduction Reiss 1980a); 
d) l'analyse des possibilités systématiques de combinaisons de traduction et de diverses formes d'exercice afin d'augmenter la compétence de traduction ;

e) la production de manuels d'enseignement de la traduction, production devant reposer sur des connaissances confirmées de la pratique de l'enseignement et de ses impératifs (pour une étude synoptique critique relative à l'allemand et à l'anglais, ou bien relative à l'allemand et au français, voir respectivement Bulmer et Glaap 1981 et Ettinger et Weller 1981);

$f$ ) en se basant sur les résultats obtenus respectivement de $a$ ) à $e$ ) l'élaboration d'une méthodologie de l'enseignement de la traduction destinée à la formation des traducteurs;

g) l'intégration d'exercices d'entraînement à la traduction dans des contextes naturels de communication;

h) comme conséquence directe de g) l'intégration de la traduction comme objectif partiel d'apprentissage (non pas comme type d'exercice) dans les directives relatives à l'enseignement des langues étrangères ;

i) la transmission de stratégies de réception de textes et de production de textes, stratégies purement spécifiques pour la traduction.

La didactique de la traduction se trouve - comme cela a été évoqué plus haut à ses débuts. Son champ d'action ne pouvait qu'être esquissé grossièrement ici. Mais il est absolument indéniable - c'est du moins mon opinion - que des polémiques de dénigrement dirigées contre le droit à l'existence d'une didactique de la traduction qui est en train de 'percer' (voir pour une telle position polémique des représentants de la perspective herméneutique Paepcke et Forget 1981 : 16f.), se trouvent dénuées de tout fondement et passent complètement à côté du champ d'action défini.

\section{LA PERSPECTIVE ORIENTÉE VERS LA TERMINOLOGIE}

Alors que la traductologie s'est pendant longtemps exclusivement préoccupée de problèmes relatifs à la théorie de la traduction ou bien de problèmes chacun respectivement concerné par la spécificité propre à deux langues, les problèmes relatifs aux langues de spécialité ont, eux, longtemps souffert de ne pas être étudiés de manière systématique. Ceci est d'autant plus étonnant que, d'une part, la pratique professionnelle du traducteur comprend des traductions en langue de spécialité comme partie intégrante originale et que, d'autre part, des réflexions d'ordre scientifiquement linguistique mais étrangères à la traduction, réflexions relatives à la langue de spécialité, se trouvaient depuis longtemps à l'ordre du jour des préoccupations scientifiques et le sont encore également aujourd'hui (à titre d'exemple, se référer aux travaux - rédigés chacun dans une optique toute différente - de l'Autrichien Wüster 1974, 1979 ; de Fluck 1980 ; du chercheur est-allemand Hoffmann 1976, 1982 ; de Drozd et Seibicke 1973; d'Ischreyt 1965 ainsi qu'aux contributions contenues dans le recueil de Bausch et al. 1978). En outre, les glossaires centrés sur des thèmes et paraissant régulièrement dans la revue Lebende Sprachen témoignent du besoin du traducteur en informations terminologiques nouvelles et complémentaires.

Il est difficile de constater à partir de quel moment le rapport entre langue de spécialité et traduction a été également 'découvert' du côté de la théorie de la traduction ; une chose est certaine : les travaux de Wüster - son travail de doctorat Internationale Sprachnormung in der Technik, besonders der Elektrotechnik (la création d'une norme linguistique internationale dans le domaine de la technique et tout particulièrement dans celui de l'électrotechnique) a été publié dès 1931 - ont exercé une certaine influence sur la naissance d'institutions de terminologie et de banques de terminologie, ainsi que la mise sur pied de dictionnaires spécialisés. Par contre, des études systématiques relatives aux rapports existant entre la langue de spécialité et la traduction sont de 
date plus récente. À ce sujet je renvoie à l'article de Wilss (1979) (pour ce qui est de l'approche théorique) et à celui de Hohnhold (1979) pour ce qui est de l'approche pratique) ainsi qu'à ceux de Arntz et Picht (1982) intitulés Einführung in die übersetzungsbezogene Terminologiearbeit (Introduction au travail terminologique relatif à la traduction). Dans ce dernier ouvrage, précisément, il est démontré en s'appuyant sur une description de la théorie de la terminologie que, dans le domaine du vocabulaire spécialisé tout particulièrement, une initiation ou formation dans la spécialité est absolument indispensable de la part du traducteur. Par 'travail de terminologie orienté vers la traduction' on entend l'aptitude du traducteur à produire 'des traductions spécialisées comme textes en langue de spécialité dans le contexte adéquat dans la langue d'arrivée' (Hohnhold $1982: 4$ ). Il faut retenir qu'avec cette notion on englobe aussi bien un domaine de recherche que l'on peut retrouver dans des dictionnaires spécialisés ou dans des glossaires de langue de spécialité (voir la contribution de Wüster 1975 à ce sujet) qu'une façon de procéder systématique du traducteur. Pour lui cela consiste à identifier comme telle une 'notion', une 'appellation', 'un mot de spécialité' ou bien une locution typique pour une langue de spécialité, à rendre chaque fois la valeur de son contenu dans le cadre d'un texte ou dans le contexte spécialisé et à chercher un équivalent adéquat dans la langue d'arrivée. Étant donné que, la plupart du temps, ceci n'est pas réalisable si l'on s'appuie uniquement sur des 'connaissances générales', le traducteur doit se spécialiser dans une large mesure pour tout ce qui, dans la matière en question appartient aux connaissances non purement linguistiques. Ce n'est que sur une telle base de connaissances qu'il est alors possible de traduire de manière satisfaisante un texte en langue de spécialités. Tant l'acquisition de nouvelles connaissances paralinguistiques que l'impossibilité relativement fréquente de pouvoir, pour le traducteur, traduire de manière adéquate ces nouvelles connaissances avec le matériel linguistique dont il dispose, rendent impératif un travail de coordination dans le domaine de la terminologie. Pour les pays de langue allemande c'est l'Institut de création des normes à Berlin ainsi que le Centre international d'information pour la terminologie à Vienne qui se sont attachés à cette tâche. Leurs travaux montrent que sans une coordination dans ce domaine, le traducteur compétent pour les langues de spécialité se trouverait fréquemment confronté à des problèmes insolubles. Mais, simultanément, la preuve de la particularité propre à la traduction de textes spécialisés face à la traduction de textes généraux est ainsi apportée. Et c'est là une différenciation qui ne devrait pas rester sans répercussion sur la didactique de la traduction.

\section{LES PERSPECTIVES 'TECHNOLOGIQUES'}

Une euphorie générale à propos des possibilités techniques de la traduction automatique dans les années cinquante et au début des années soixante fit place par la suite à une attitude plus réaliste. $\mathrm{Si}$, au départ, on croyait encore pouvoir un jour faire presque entièrement traduire un texte par une machine, les espoirs ultérieurs se réduisirent considérablement. Certains adoptèrent une limitation consciente et voulue à des types de texte présentant une structure relativement simple, en partie hautement standardisés (par exemple Brockhaus 1971), d'autres envisagèrent plutôt l'utilisation de la machine simplement comme soutien pour la traduction effective, non plus pour la supplanter totalement (par exemple Krollmann 1981). Et, en effet, les travaux présentant une vue d'ensemble (voir par exemple Bruderer 1979 ainsi que Wilss 1977 : 297ff. et 1985) confirment que même les nouvelles évolutions dans le domaine de la linguistique tant que dans celui de la technique ne sont en mesure ni de maîtriser de manière adéquate la complexité des langues naturelles ni l'utilisation du langage humain, ni de la transposer dans un programme adéquat (voir aussi Freigang 1984). Aussi n'est-il pas étonnant que Wilss (1977 : 322) ne donne aucune chance à la mise au point d'algorithmes de traduction dé- 
nués de contexte (autre est la position représentée par Blatt 1984), mais qu'il considère par contre comme étant tout à fait réalisable " de dégager d'après des critères de fréquence des régularités structurelles et d'en déduire des régularités de transposition susceptibles d'être traitées par la machine ". En effet, à l'inverse du traducteur pour qui la réception du texte de départ et le processus de traduction s'effectuent de façon individuelle et sélective (voir ci-dessus), la machine est uniquement en mesure d'exécuter, en fonction d'une programmation établie, les opérations ainsi requises - et elles, uniquement - et ce, dans un ordre préalablement fixé. Alors que, ce faisant, elle présente, à titre de traducteur, de vastes lacunes ${ }^{6}$ et est même susceptible de représenter un réel danger à titre de 'conseiller' dans le cadre de réflexions relatives à la didactique de la traduction, elle se révèle être, par contre, extrêmement efficace pour le travail dans le domaine de la terminologie, et ne peut plus être ignorée dans la pratique quotidienne des traducteurs spécialisés travaillant dans les grandes institutions (cf. Arntz et Picht 1982 : 196 et aussi Freigang 1984). Il n'y a, pour cette raison, rien d'étonnant à ce que l'on se détourne de la traduction automatique pour se tourner alors vers une traduction assistée par la machine et pour laquelle le traducteur reste la condition sine qua non en tant qu'instance souveraine, instance de décision et instance de réalisation (voir Krollmann 1981 et aussi Schmitz 1984). C'est sur cet arrière-plan qu'il faut situer la tentative entreprise dans le cadre du domaine de recherche spéciale de Sarrebruck répondant à l'appellation 'recherche linguistique électronique', tentative consistant à relier entre elles différentes théories linguistiques (ici : la grammaire générative et la grammaire valencielle) lors de l'élaboration de programmes, pour pouvoir, à partir des résultats ainsi obtenus, s'orienter vers une traduction assistée par la machine, traduction assistée dont la compétence ne se limite plus uniquement à établir des listes de correspondances standard du type 1:1. Les travaux entrepris à cet effet sont actuellement en cours (cf. Wilss 1985).

\section{PERSPECTIVES D'AVENIR}

La traductologie est encore loin d'avoir résolu tous les problèmes qui se posent à elles - vraisemblablement n'a-t-elle même pas encore repéré tous les problèmes. À ceci vient s'ajouter son caractère quasiment kaléidoscopique qui, bien entendu, ne facilite en rien l'élaboration d'une théorie fondamentale. Ce n'est pas un hasard si la tentative visant à créer une théorie générale de la traduction ne date que de 1984 (voir Reiss et Vermeer 1984). Outre les perspectives intéressant directement la traductologie et mentionnées dans cet article, il existe toute une série de perspectives qui en découlent ou qui s'en laissent déduire de manière plus ou moins directe. Qu'il me soit permis de citer à titre d'exemple les différents aspects touchant à la lexicographie (par exemple Hausmann 1977 ; Haensch et al. 1982 ; Werner 1982 ; le recueil d'articles de Götz et Herbst 1984), à l'apprentissage du vocabulaire (cf. les diverses orientations chez Müller 1981 et Hausmann 1984), à la textologie contrastive (par exemple Hartmann 1981 ; Spillner 1981) et ainsi qu'attenant à la linguistique du discours (cf. à ce sujet quelques contributions dans le recueil de House-Edmondson et Blum-Kulka 1985). Dans quelle mesure chacune de ces perspectives est à même de répondre aux attentes et aux espoirs mis en elle ? C'est là une question dont la réponse reste actuellement en suspens. Toutefois, il se dessine clairement que la traductologie envisage de franchir le pas qui, d'une science au départ orientée vers une approche linguistique à caractère statique, fera d'elle une science concernée directement par l'activité même de traduire et orientée vers le processus mis en œuvre et les résultats ainsi obtenus. Pour ce qui est des autres méthodes et résultats de la recherche, il y a lieu d'être dans l'expectative, ne serait-ce qu'en raison des conséquences qu'ils impliquent pour la formation, entre autres, des interprètes et traducteurs. 
Il se peut que Martin Luther ait apporté beaucoup de choses aux Allemands mais - Dieu soit loué - en ce qui concerne la traduction son point de vue est dépassé (voir la citation introductive).

Notes

* Marie-Elisabeth Parent a eu l'amabilité de bien vouloir m'aider afin de me permettre de publier en français le texte de cet article, ce dont je tiens tout particulièrement à la remercier ici.

1. Le livre de Hönig et Kussmaul (1982) dans lequel les auteurs entreprennent à titre d'essai de relier directement des éléments théoriques à une pratique concrète de la traduction, fait ici figure d'exception et même d'une exception tout à fait méritoire.

2. Je renvoie ici le lecteur à une acception de la didactique assez confuse que Paepcke et Forget $(1981: 16)$ mettent à jour et qui leur bloque l'accès à des approches orientées différemment. Une analyse critique de la perspective herméneutique se trouve contenue dans la recension de Reiss (1984).

3. Hönig et Kussmaul (1982) parlent à ce sujet de stratégie de la traduction. Étant donné qu'ils désignent par là un procédé qualitativement autre que celui dont il est question dans la discussion sur la stratégie en psycholinguistique (cf. à ce sujet Faerch et Kasper 1983 par exemple), je considère le terme de 'procédé' pour mieux approprié à ce que veulent dire Hönig et Kussmaul (cf. Königs 1985a).

4. Il faut remarquer à ce sujet que cette tradition n'a jamais divulgué ses résultats sous l'étiquette de 'didactique de la traduction' mais, la plupart du temps, dans le cadre des conceptions 'pédagogiques', voire même relatives à la didactique de l'enseignement des langues.

5. Arntz et Thiel (1981) ont montré comment un tel procédé peut être enseigné à des futurs traducteurs, et ce, dans le cadre du déroulement de leurs études.

6. Indépendamment de textes hautement standardisés comme c'est le cas du système de Sarrebruck SUSYBSA.

\section{BIBLIOGRAPHIE}

ALBRECHT, J. (1973) : Linguistik und Übersetzung, Tübingen, Niemeyer.

ARNTZ, R. und H. PICHT (1982) : Einführung in die iubersetzungsbezogene Terminologiearbeit, Hildesheim, Olms.

ARNZT, R. und G. THIEL (1981) : " Das terminologische Seminar in der Übersetzerausbildung ", in : W. Kühlwein und A. Raasch (eds.), Sprache : Lehren - Lernen. Band 1, Kongressberichte der 11, Jahrestagung der Gesellschaft für Angewandte Linguistik, Tübingen, Narr, pp. 57-63.

BBAUSCH, K.-R. (1970) : "Übersetzungswissenschaft und angewandte Sprachwissenschaft. Versuch einer Standortbestimmung ", Lebende Sprachen 15, pp. 161-163.

BAUSCH, K.-R. (1977a) : "Perspektiven für die Analyse von Übersetzungsunterricht ", in : K.-H. Bender, K. Berger und M. Wandruszka (eds.), Imago Linguae. Beiträge zu Sprache, Deutung und Übersetzen. Fetschrift zum 60. Geburtstag von Fritz Paepcke. München, Fink, pp. 29-33.

BAUSCH, K.-R. (1977b) : "Zur Übertragung der 'Übersetzung als Fertigkeit' auf die 'Übersetzung als Übungsform' ", Die Neueren Sprachen 5/6, pp. 517-535.

BAUSCH, K.-R. (1979) : "Die Erstellung von Didaktischen Grammatiken als Exempel für das Verhältnis von Angewandter Linguistik, Fremdsprachendidaktik und Sprachlehrforschung", in : K.-R. Bausch (ed.), Beiträge zur Didaktischen Grammatik. Probleme, Konzepte, Beispiele, Königstein, Scriptor, pp. 2-24.

BAUSCH, K.-R., U. BLIESENER, H. CHRIST, K. SCHRÖDER, und U. WEISBROD (eds.) (1978) : Beiträge zum Verhältnis von Faschsprache und Gemeinsprache im Fremdsprachenunterricht der Sekundarstufe II, Bochum, Seminar für Sprachlehrforschung/Heidelberg, Groos.

BAUSCH, K.-R. und F.-R. WELLER (eds.) (1981) : Übersetzen und Fremdsprachenunterricht, Frankfurt, Diesterweg.

DE BEAUGRANDE, R. und W.U. DRESSLER (1981) : Einführung in die Textlinguistik, Tübingen, Niemeyer.

BLATT, A. (1984); " Erarbeitung algorithmischer Vorgehensweisen in der Übersetzerausbildung ", in : W. Wilss and G. Thome (eds.), Die Theorie des Übersetzens und ihr Aufschlusswert für die Übersetzungs- und Dolmetschdidaktik. Translation Theory and its Implementation in the Teaching of Translating and Interpreting, Tübingen, Narr, pp. 356-360.

BROCKHAUS, K. (1971) : Automatische Übersetzung. Untersuchungen am Beispiel der Sprachen English und Deutsch, Braunschweig, Vieweg.

BRUDERER, H.E. (1978) : Handbuch der maschinellen und maschinenunterstützten Sprachübersetzung. Automatische Übersetzung natürlicher Sprachen und Mehrsprachige Terminologiedatenbanken, München/New York, Verlag Dokumentation. 
BULMER, A.F. und A.-R. GLAAP (1981): « Kritische Synopse von Übersetzungsbüchern - English », in : K.-R. Bausch and F.-R. Weller (eds.), Übersetzen und Fremdsprachenunterricht, Frankfurt, Kiesterweg, pp. 156-172.

DILLER, H.-J. und J. KORNELIUS (1978) : Linguistische Probleme der Übersetzung, Tübingen, Niemeyer. DRESSLER, W. (1972a) : Einführung in die Textlinguistik, Tübingen, Niemeyer.

DRESSLER, W. (1972b) : " Textgrammatische Invarianz in Übersetzungen ? ", in : E. Gülich and W. Raible (eds.), Textsorten, Differenzierungskriterien aus linguistischer Sicht, Frankfurt, Athenäum, pp. 98-106.

DROZD, L. und W. SEIBICKE (1973) : Deutsche Fach- und Wissenschaftssprache. Bestandsaufnahme, Theorie, Geschichte, Wiesbaden, Brandstetter.

ERICSSON, K.A. und H.A. SIMON (1984) : Protocol Analysis. Verbal Reports as Data, Cambridge, Mass., MIT Press.

ETTINGER, S. und F.-R. WELLER (1981) : "Kritische Synopse von Übersetzungsbüchern Französisch ", in : K.-R. Bausch und F.-R. Weller (eds.), Übersetzen und Fremdsprachenunterricht, Frankfurt, Diesterweg, pp. 173-191.

FAERCH, K. und G. KASPER (eds.) (1983) : Strategies in Interlanguage Communication, London/New York, Longman.

FLUCK, H.-R. (1980) : Fachsprachen, München, Francke, $2^{\mathrm{e}}$ édition.

FREIGANG, K.-H. (1984) : "Maschinelle und maschinengestïtzte Übersetzungsverfahren in der Praxis ", in : W. Wilss und G. Thome (eds.), Die Theorie des Übersetzens und ihr Aufschlusswert für die Übersetzungs- und Dolmetschdidaktik. Translation Theory and its Implementation in the Teaching of Translating and Interpreting, Tübingen, Narr, pp. 344-349.

GÖTZ, D. und T. HERBST (eds.) (1984) : Theoretische und praktische Probleme der Lexikographie, (1. Augsburger Kolloquium vom 29-30.4.1983), München, Hueber.

GROTJAHN, R. und U. KLEVINGHAUS (1975) : Zum Stellenwert der Übersetzung im Fremdsprachenunterricht. Eine Pilotstudie, Bochum, Seminar für Sprachlehrforschung/Heidelberg, Groos.

G. HAENSCH, L. WOLF, S. ETTINGER und R. WERNER (1983) : La Lexiocografia. De la Lingïistica a la Lexicografia práctica, Madrid, Gredos.

HARTMANN, R.R.K. (1981) : " Contrastive Textology and Translation ", in : W. Kühlwein, G. Thome und W. Wilss (eds.), Kontrastive Linguistik und Ubersetzungswissenschaft. Akten des Internationalen Kolloquiums Trier/Saarbrücken 25-30.4.1978, München, Fink, pp. 200-208.

HAUSMANN, F.J. (1977) : Einführung in die Benutzung der neufranzösischen Wörterbüicher, Tübingen, Niemeyer.

HAUSMANN, F.J. (1984) : "Wortschatzlernen ist Kollokationslernen. Zum Lehren und Lernen Französischer Wortverbindungen ", Praxis des Neusprachlichen Unterrichts 4, pp. 395-406.

HÖNIG, H.G. und P. KUSSMAUL (1982) : Strategie der Übersetzung. Ein Lehr- und Arbeitsbuch, Tübingen, Narr.

HOFFMANN, L. (1976) : Kommunikationsmittel Fachsprache. Eine Einführung, Berlin, Akademie-Verlag.

HOFFMANN, L. (1982) : "Probleme und Methoden der Fachsprachenforschung ", in : J.R. Richard, G. Thome und W. Wilss (eds.), Fachsprachenforschung und -lehre. Schwerpunkt Spanisch. Internationales Kolloquium an der Universität des Saarlandes, Saarbrü̈cken, 6-8.11.1980, Tübingen, Narr, pp. 1-13.

HOHNHOLD, I. (1979) : "Übersetzungsorientierte Terminologiearbeit - fachsprachlich orientiertes Übersetzen ", Lebende Sprachen 4, pp. 153-156.

HOHNHOLD, I. (1982) : "Grundbegriffe im Bereich und im Umfeld Übersetzungsorientierter Terminologiearbeit. Arbeitsdefinitionen und Anmerkungen ", Lebende Sprachen 1, pp. 1-5.

HOUSE, J. (1977) : A Model for Translation Quality Assessment, Tübingen, Narr.

HOUSE-EDMONDSON, J. and S. BLUM-KULKA (eds.) (1985) : Interlingual and Intercultural Communication : Discourse and Cognition in Translation, Tübingen, Narr.

HUBER, G.L., und H. MANDL (eds.) (1982) : Verbal Daten. Eine Einführung in Die Grundlagen und Methoden der Erhebung und Auswertung, Weinheim/Basel, Beltz.

ISCHREYT, H. (1965) : Studien zum Verhältnis von Sprache und Technik. Institutionelle Sprachlenkung in der Terminologie der Technik, Düsseldorf, Schwann.

KöNIGS, F.G. (1979) : Übersetzung in Theorie und Praxis : Ansatzpunkte für die Konzeption einer Didaktik der Übersetzung, Bochum, Seminar für Sprachlehrforschung/Heidelberg, Groos.

KÖNIGS, F.G. (1981a) : "Übersetzung und fremdsprachenunterricht - vereinbar oder unvereinbar ? ", in K.-R. Bausch und F.-R. Weller (eds.), Übersetzen und Fremdsprachenunterricht, Frankfurt, Diesterweg, pp. 203-216.

KÖNIGS, F.G. (1981b) : "Zur Frage der Übersetzungseinheit und ihre Relevanz für den Fremdsprachenunterricht ", Linguistische Berichte 74, pp. 82-103.

KÖNIGS, F.G. (1985a) : «Der Vorgang des Übersetzens : Theoretische Modelle und Praktischer Vollzug. Zum Verhältnis von Theorie und Praxis in der Übersetzungswissenschaft ", Lebende Sprachen 4. 
KÖNIGS, F.G. (1985b) : "Translation Inside and Outside the Teaching Context : the Text as a Starting Point ", in : C. Titford and A.E. Hieke (eds.), Translation in Foreign Language Teaching and Testing, Tübingen, Narr, pp. 29-48.

KÖNIGS, F.G. (1986 : à paraître) : "Was beim Übersetzen Passiert. Theoretische Aspekte, Empirische Befunde und Praktische Konsequenzen ", Die Neueren Sprachen.

KOLLER, W. (1978) : «Kritik der Theorie der Übersetzungskritik », IRAL 2, pp. 89-107.

KOLLER, W. (1979) : Einführung in die Übersetzungswissenschaft, Heidelberg, Quelle \& Meyer.

KRINGS, H.P. (1985) : "Translation Problems and Translation Strategies of Advanced German Learners of French (L2)", in : J. House-Edmondson and S. Blum-Kulka (eds.), Interlingual and Intercultural Communication : Discourse and Cognition in Translation, Tübingen, Narr.

KRINGS, H.P. (en préparation) : Übersetzungsprozess und Übersetzungsdidaktik.

KROLLMANN, F. (1981) : " Hilft der Computer dem Übersetzer oder Vernichtet er seinen Arbeitsplatz ? », in : LANGENSCHEIDT-VERLAG (ed.), Wort und Sprache. Beiträge zu Problemen der Lexikographie und Sprachpraxis, Berlin/München/Wien/Zürich, Langenscheidt, pp. 13-23.

LAROSE, R. (1984) : Paramètres d'évaluation des traductions : théories contemporaines et approche textologique, Université de Montréal (thèse de doctorat).

MALBLANC, A. (1968) : Stylistique comparée du français et de l'allemand, Paris, Didier, $4^{e}$ édition.

MÜLLER, B.-D. (1981) : "Bedeutungserwerb - ein Lernprozess in Etappen ", in : B.-D. Müller (ed.) Konfrontative Semantik, Zur Kritik am Fremdsprachenunterricht aus Grundlagentheoretischer Sicht Kritischer Psychologie und Sozialwissenschaftlicher Linguistik, Tübingen, Narr/Weil der Stadt, Lexika, pp. 113-154.

PAEPCKE, F. und P. FORGET (1981) : Textverstehen und Übersetzen. Ouvertures sur la traduction, Heidelberg, Groos.

RAABE, H. (1979) : "Didaktische Translationsgrammatik : Aspekte einer auf den Erwerb von Ïbersetzungsfähigkeit Ausgerichteten Grammatik», in : K.-R. Bausch (ed.), Beiträge zur Didaktischen Grammatik. Probleme, Konzepte, Beispiele, Königstein, Scriptor, pp. 239-256.

REISS, K. (1971) : Möglichkeiten und Grenzen der Übersetzungskritik, München, Hueber.

REISS, K. (1976) : Texttyp und Übersetzungsmethode. Der Operative Text, Kronberg, Scriptor.

REISS, K. (1977) : "Textsortenkonventionen. Vergleichende Untersuchungen zur Todesanzeige", le Langage et l'homme 35, pp. 46-54.

REISS, K. (1978a) : "Anwendbarkeit der Texttypologie mit Besonderer Berücksichtigung der Sachprosa ", in : K. Gomard and S.O. Poulsen (eds.), Übersetzungswissenschaft, Arthus, Université, pp. 27-35.

REISS, K. (1978b) : "Textsortenkonventionen. Vergleichende Untersuchungen zur Todesanzeige", le Langage et l'homme $36, \mathrm{pp} .60-68$ (deuxième partie).

REISS, K. (1979) : "Übersetzen als Semiotisch Bestimmte Transferoperation ", in : W. Kühlwein and A. Raasch (eds.), Kongressbericht der 9. Jahrestagung der Gesellschaft für Angewandte Linguistik, Heidelberg, Gross, pp. 1-10.

REISS, K. (1980a) : " Der Übersetzungsvergleich als Didaktisches Instrument im Übersetzungsunterricht ", in : S.-O. Poulsen and W. Wilss (eds.), Angewandte Übersetzungswissenschaft. Internationales Übersetzungswissenschaftliches Kolloquium an der Wirtschaftuniversität Arhus, Arhus, Université, pp. 149-164.

REISS, K. (1980b) : "Que Dios nos Coja Confesados! Zur Problematik Pragmatischer Divergenzen bein Übersetzen aus dem Spanischen ins Deutsche", in : H.D. Bork, A. Greive und D. Woll (eds.), Romanica Europaea et Americana. Festschrift für Harri Meier, Bonn, Bouvier, pp. 463-472.

REISS, K. (1984) : "Compte rendu : F. Paepcke et P. Forget, TEXTVERSTEHEN UND ÜBERSETZEN. OUVERTURES SUR LA TRADUCTION. Heidelberg, Groos 1981 ", Die Neueren Sprachen 4, pp. 480-482.

REISS, K. und H.J. VERMEER (1984) : Grundlegung einer Allgemeinen Translationstheorie, Tübingen, Niemeyer.

SCHMITZ, K.-D. (1984) : "Automation im Umfeld des Übersetzens und deren Berücksichtigung im Rahmen der Übersetzerausbildung ", in : W. Wilss and G. Thome (eds.), Die Theorie des Übersetzens und ihr Aufschlusswert für die Übersetzungs- und Dolmetschdidaktik. Translation Theory and its Implementation in the Teaching of Translating and Interpreting, Tübingen, Narr, pp. 350-355.

SPILLNER, B. (1981) : "Textsorten im Sprachvergleich. Ansätze zu einer Kontrastiven Textologie ", in : W. Kühlwein, G. Thome and W. Wilss (eds.), Kontrastive Linguistik und Übersetzungswissenschaft. Akten des Internationalen Kolloquiums Trier/Saarbrïcken 25-30.9.1978, München, Fink, pp. 239-250.

STEIN, D. (1980) : Theoretische Grundlagen der Übersetzungswissenschaft, Tübingen, Narr.

STOLZE, R. (1982) : Grundlagen der Textübersetzung, Heidelberg, Groos.

THIEL, G. (1974) : « Ansätze zu einer Texttypologie der Übersetzungsrelevanten Textanalyse », in : V. Kapp (ed.), Übersetzer und Dolmetscher. Theoretische Grundlagen, Ausbildung, Berufspraxis, Heidelberg, Quelle \& Meyer, pp. 174-185. 
THIEL, G. (1981) : «Überlegungen zur Übersetzungrelevanten Textanalyse »; in W. Wilss (ed.) : Übersetzungswissenschaft, Darmstadt, Wissenschaftliche Buchgesellschaft, pp. 367-383.

TITFORD, C. und A.E. HIEKE (eds.) (1985) : Translation in Foreign Language Teaching and Testing, Tübingen, Narr.

VINAY, J.-P. et J. DARBELNET (1958) : Stylistique comparée du français et de l'anglais. Méthode de traduction, Paris, Didier.

WAGNER, A.C., S. MAIER, I. UTTENDORFER-MAREK und R.H. WEIDLE (1981) : Unterrichtspsychogramme. Was in den Köpfen von Lehrern und Schülern Vorgeht. Zugang zur Subjektiven Unterrichtswirklichkeit, Reinbek, rororo.

WELLER, F.-R. (1977) : "Auswahlbibliographie zur Ubersetzung im Fremdsprachenunterricht ", Die Neueren Sprachen 5/6, pp. 588-603.

WELLER, F.-R. (1981) : " Formen und Funktionen des Übersetzens im Fremdsprachenunterricht. Aspekte einer Schulischen Übersetzungslehre am Beispeil des Französischen ", in : K.-R. Bausch und F.-R. Weller (eds.), Übersetzen und Fremdsprachenunterricht, Frankfurt, Diesterweg, pp. 233-296.

WERNER, r. (1982) : "Zur Reihenfolge der Definitionen bzw. Ubersetzungsäquivalente im Wörterbuchartikel (mit besonderer Berücksichtigung Spanischer Beispiele) ", Lebende Sprachen 4, pp. $150-156$.

WILSS, W. (1975) : " Möglichkeiten und Grenzen der Didaktisierbarkeit des Übersetzungsunterrichts », in : W. Wilss (ed.), Kongressbericht der 6. Jahrestagung der Gesellschaft für Angewandte Linguistik. Band 1 : Übersetzungswissenschaft, Heidelberg, Groos, pp. 1-23.

WILSS, W. (1977) : Übersetzungswissenschaft - Probleme und Methoden, Stuttgart, Klett.

WILSS, W. (1978) : "Vorüberlegungen zu einer Didaktischen Grammatik des Übersetzens ", Lebende Sprachen 4, pp. 145-151.

WILSS, W. (1979) : "Fachsprache und Übersetzen", in H. Felber, F. Lang und G. Wersig (eds.) : Terminologie als Angewandte Sprachwissenschaft. Gedenkschrift für Eugen Wüster, München/Paris, Saur, pp. 177-191.

WILSS, W. (1980) : "Semiotik und Übersetzungswissenschaft ", in : W. Wilss (ed.), Semiotik und Übersetzen, Tübingen, Narr, pp. 9-22.

WILSS, W. (1981) : "Einleitung", in : W. Wilss (ed.), Übersetzungswissenschaft, Darmstadt, Wissenschaftliche Buchgesellschaft, pp. 1-23.

WILSS, W. (1983) : "Translation Strategy, Translation Method and Translation Technique : Towards a Clarification of Three Translational Concepts ", Revue de phonétique appliquée 66-68, pp. 143-152.

WILSS, W. (1985) : "Menschliche Übersetzung und Maschinelle Übersetzung ", Lebende Sprachen 1, pp. $10-15$.

WILSS, W. und G. THOME (eds.) (1974a) : Aspekte der Theoretischen, Sprachenpaarbezogenen und Angewandten Sprachwissenschaft. Referate und Diskussionsbeiträge des 1. übersetzungswissenschaftlichen Kolloquiums am Institut für Übersetzen und Dolmetschen der Universität des Saarlandes (26-27.5.1972). Saarbrüicken, Université.

WILSS, W. und G. THOME (eds.) (1974b) : Aspekte der Theoretischen, Sprachenpaarbezogenen une Angewandten Übersetzungswissenschaft II, Heidelberg, Groos.

WILSS, W. und G. THOME (eds.) (1984) : Die Theorie des Übersetzens und ihr Aufschlusswert für die Übersetzungs- und Dolmetschdidaktik. Translation Theory and Its Implementation in the Teaching of Translating and Interpreting, Tübingen, Narr.

WÜSTER, E. (1974) : « Die Allgemeine Terminologielehre - ein Grenzgebiet Zwischen Sprachwissenschaft, Logik, Ontologie, Informatik und den Sachwissenschaften ", Linguistics 119, pp. 61-106.

WÜSTER, E. (1975) : "Die Ausbildung in Terminologie und Terminologischer Lexikographie ", Lebende Sprachen 2, pp. 33-37.

WÜSTER, E. (1979) : Einführung in die Allgemeine Terminologielehre und Terminologische Lexikographie, Wien/New York, Springer. 